\title{
El marco conceptual del sistema nacional de innovación y las economías menos desarrolladas
}

\section{The Conceptual Frame of the National Invention System and the Less-Developed Economies}

Ancelmo José Vega Armenta*

Artículo de reflexión

Cómo citar este artículo: Vega Armenta, A. J. (2011). El marco conceptual del sistema nacional de innovación y las economías menos desarrolladas. Revista CIFE, 17, (12), 151 - 166.

\section{Resumen}

El fenómeno de la innovación en los países de menor desarrollo industrial es un tema difícil desde el punto de vista del tratamiento analítico y en relación con la formulación de políticas públicas para su promoción. En ambos casos se identifica un sesgo en favor del estudio de los fenómenos de transferencia y difusión tecnológica generados en las economías industrializadas, que se recibe pasivamente en los menos industrializados, en detrimento del interés analítico por otros temas significativos, entre ellos la manera como las economías de menor desarrollo industrial tienen la habilidad para generar, apropiar y usar conocimiento con fines productivos.

En este artículo se discute qué tan adecuada es la teoría del Sistema Nacional de Innovación (NSI, por sus siglas en inglés) como un marco conceptual y metodológico, en la perspectiva neo-schumpeteriana y evolucionista, para el estudio de la innovación en el contexto de los países de menor desarrollo industrial. La innovación real en estos países presenta como características sobresalientes la de-

* Ingeniero Mecánico, Magíster en Ciencias Económicas. Candidato a Doctor en Economía, Universidad Nacional de Colombia - CEIBA. Docente de la Maestría en Ciencias Económicas, Universidad Santo Tomás. Correo electrónico: <ajuegar@gmail.com>. 
bilidad de las relaciones entre los agentes en el sistema económico y, por supuesto, bajas capacidades tecnológicas y de aprendizaje productivo.

En la primera parte del artículo se discuten los principales elementos involucrados en la teoría del NSI, sus principales implicaciones y limitaciones. Seguidamente, en la segunda parte, se discuten las relaciones entre las estructuras micro-meso-macro como elemento central del marco analítico y, especialmente, metodológico, en el tratamiento del papel de las instituciones como componentes del NSI, y determinantes del desempeño innovador en las economías de menor desarrollo industrial.

Finalmente, en la tercera parte, se discuten algunas implicaciones relacionadas con la aplicación de este marco conceptual y metodológico en el estudio del desempeño institucional e innovador empresarial en estas economías

Palabras clave: economía evolucionaria, schumpeterianos, Sistema Nacional de Innovación, instituciones, metodología económica.

Glasificación JEL: B520, B40.

\section{Abstract}

Innovation is a very elusive phenomenon in less industrial economies because it is not as frequently encountered as in industrialized ones. Moreover it is difficult to analyse and to promote.

As a consequence, there is a bias which favours the analysis of transference and diffusion of technology and knowledge coming from the industrialized countries in detriment of other interesting and significant topics, sush as the ways the less industrialized economies are able to generate, appropriate and use productive knowledge.

This article addresses the suitableness of National System of Innovation (NSI), as an analytical and methodological framework, in the neo-schumpeterian and evolutionary perspective, to deal the innovation in the context of lessindustrialized economies. The real innovation activity in these countries is characterized by weak relationships between economic agents and low technological capabilities and capacity for productive learning.

The first part of the article discusses NSI as a conceptual framework, its main implications and the necessity of transforming some of its aspects in the context of less industrialized countries. Furthermore, in the second part, the relationship between micro-meso-macro structures are discussed as the methodological framework core in addressing the rol of institutions as an NSI component and as a determinant of innovative performance in less industrialized economies.

Finally, as a conclusion, in the third part of the article, some specific analytical and methodological issues related to applications in the study of innovation performance in less industrialized economies are discussed.

Key words: Evolutionary Economic, Schumpeterian, National System of Innovation, Institution, Economic Methodology.

JEL Classification: B520, B40. 


\section{Introducción}

En una evaluación crítica y propositiva de las condiciones y características de la actividad innovadora en los países de América Latina después de diez años de reformas aperturistas, Arocena y Sutz $(2000,2002)$ señalan que la principal restricción para su fortalecimiento y promoción es el bajo nivel de gasto nacional en innovación, en promedio menor que el $1 \%$ del PIB, nivel de gasto que era el valor mínimo establecido por la UNESCO tres décadas atrás.

Este hallazgo confirma el precario esfuerzo y compromiso de los gobiernos y el sector empresarial en estos países para el fortalecimiento de capacidades para la generación, apropiación y uso de conocimiento, hecho poco sorprendente, dado el secular atraso tecnológico que en ellos existe, el cual, no obstante, contrasta con otros hallazgos reportados por los autores:

1. Registros importantes, aunque no generalizados, de gastos en investigación y desarrollo (I+D) internos, dirigidos específicamente a la contratación de profesionales y técnicos por parte de empresas con desempeño innovador.

2. El carácter altamente informal de la innovación industrial. Aún en los casos en que una buena proporción de empresas industriales realizan innovación en productos y procesos, la actividad de I+D no aparece clara y formalmente articulada con la estrategia competitiva empresarial.

3. Las encuestas de innovación ${ }^{1}$ revelan que ésta no es necesariamente de bajo nivel de complejidad y, aso-

1 La realización más o menos sistemática de encuestas de innovación en América Latina es cada vez más frecuente y generalizada. En Colombia, hasta la fecha, se han realizado tres, en 1998, 2003 y 2008. ciado a ello, existe una contratación significativa de personal cualificado (profesionales) por parte de las empresas innovadoras.

4. La percepción de que las empresas consideran las ideas para innovación y actualización tecnológica como un asunto primordialmente interno.

5. Las necesidades de personal cualificado en las pequeñas y medianas empresas (PYMES) no se suplen internamente, mediante la capacitación; éstas se proveen del personal calificado mediante relaciones, aún débiles, con universidades e institutos de investigación.

6. La innovación en las empresas nacionales se basa en lazos con empresas extranjeras, especialmente a través de la adquisición de bienes de capital; de hecho, la adquisición de maquinaria y equipo se considera entre los planes de innovación de las firmas.

En su conjunto, estos hallazgos suscitan una reflexión acerca del verdadero significado y el alcance de la innovación en los países de menor desarrollo industrial, más allá de los aportes del análisis que, en la perspectiva convencional, privilegian el estudio de la difusión y la transferencia de tecnología generada en el mundo industrializado, asumiendo condiciones de igualdad respecto a la disponibilidad y accesibilidad al conocimiento y la tecnología.

Sin lugar a dudas el análisis convencional ha permitido avanzar significativamente en la identificación de ciertas características del conocimiento determinantes de las capacidades innovadoras y la competitividad de industrias y empresas en economías no industrializadas. En el nivel agregado, ha contribuido con la identificación y caracterización de brechas tecnológicas, sus consecuencias y posibles alternativas estratégicas para su superación; y, en el ámbito de la empresa, la caracterización de los procesos 
de apropiación, aprendizaje y gestión tecnológica, entre otros tópicos (Katz, 2006).

No obstante, los aportes en el tratamiento de otras temáticas relacionados con el cambio tecnológico cuya relevancia ha sido reconocida en la literatura económica reciente, tales como la generación endógena de conocimiento, implícita en los resultados arriba presentados, han sido particularmente escasos. En este sentido, puede afirmarse que el análisis del problema de la producción y transferencia de conocimiento no se ha abordado convenientemente, dificultando tanto la comprensión del fenómeno como la eficacia en la formulación de políticas.

De acuerdo con Arocena y Sutz (2006), el atraso tecnológico de la mayoría de los países, persistente en la economía globalizada actual, requiere de una aproximación analítica y metodológica también actualizada que aborde cuestiones relevantes como los procesos de innovación, entendida como la generación, uso y apropiación de conocimiento en las condiciones de la periferia capitalista.

En este artículo se argumenta que, con algunos ajustes, el marco conceptual del Sistema Nacional de Innovación (NSI) junto a los avances recientes contenidos en la denominada estructura analítica micro-meso-macro, brindan posibilidades analíticas y metodológicas adecuadas para avanzar en el estudio de los mecanismos por los cuales la dinámica institucional tiene incidencia en la actividad innovadora y el cambio tecnológico en el contexto de las economías no industrializadas.

Desde la perspectiva analítica y metodológica adoptada en este trabajo, se evidencian ciertas limitaciones de la teoría convencional, especialmente significativas para los estudios sobre innovación y cambio tecnológico en los países de América Latina. De manera específica, se destacan algunas limitaciones metodológicas y analíticas impuestas por el supuesto carácter exógeno del conocimiento que, en lo fundamental, no permiten identificar ni caracterizar la actividad innovadora realmente existente en ellas.

La diferencia sustancial del enfoque analítico esbozado en este artículo consiste, en primer lugar, en el rescate del concepto schumpeteriano ${ }^{2}$ de innovación, no restringido al avance tecnológico formal expresado en el número de patentes y gastos en investigación y desarrollo; y, en segundo término, la posibilidad de la inclusión analítica de la diversidad de las relaciones entre agentes del sistema económico como punto de partida del surgimiento y transformación de las instituciones cuyo funcionamiento repercute de manera directa en el desempeño innovador de las empresas.

La visión schumpeteriana del proceso de creación-destrucción subyacente al concepto del NSI supone una dinámica de ganadores y perdedores; de este modo, el eje central de la argumentación es la hipótesis de que la innovación confiere ventajas decisivas en términos de costos o calidad y determina no sólo los márgenes de beneficios, sino las posibilidades de supervivencia y supremacía de las empresas (Williamson, 1975).

En síntesis, el marco conceptual del NSI brinda una alternativa analítica que permitiría abordar el análisis del fenómeno de la innovación y el proceso de generación y aplicación de conocimiento en la producción, asumiendo el comportamiento sistémico de la economía expresado en un conjunto estable e institucionalizado de relaciones entre agentes económicos (empresas, entidades de gobierno y educación, entre otros). Las dinámicas innova-

2 La innovación consiste en la introducción de un nuevo bien, un nuevo método de producción, la apertura de un nuevo mercado, la conquista de una nueva fuente de abastecimiento de insumos y nuevas formas de organización en cualquier industria, como la creación de una posición monopolista (Schumpeter, 1989, p. 66). 
doras se abordan analíticamente en un contexto nacional específico, en condiciones concretas de relaciones sociales, económicas, políticas y culturales (Sutz, 1998), en las cuales las empresas, enfrentadas a las mismas señales del mercado, responden de modos diferenciados en procura de mejores resultados (innovaciones) y compiten incentivadas por la obtención de ganancias, actuales o prospectivas. El mercado es uno de los mecanismos que, además de asignar recursos, sirve para dirigir y evaluar la búsqueda de nuevas alternativas de producción u organización.

Además de esta introducción, en la primera parte se aborda la discusión respecto al análisis del fenómeno de la innovación y el cambio tecnológico en la perspectiva del marco conceptual del NSI que pone el énfasis en el papel de las instituciones. En la segunda parte se describen algunas similitudes y coincidencias entre la teoría de los NSI y la estructura micro-meso-macro que sirven de sustento a la discusión sobre la propuesta analítica y metodológica.

Por último, a manera de conclusión, en la tercera parte se discuten algunas de las bondades en la aplicación de esta estructura analítica al estudio de las interacciones entre agentes como determinantes de la innovación empresarial en economías de menor desarrollo.

\section{El NSI, las instituciones y capacidades innovadoras en economías no industrializadas}

De acuerdo con Arocena y Sutz (2002), el marco conceptual del $\mathrm{NSI}^{3}$, con ciertas adecuaciones, es una herramienta útil en la reconsideración del problema del

3 Más que una teoría formal, en el sentido de contener proposiciones respecto a relaciones entre variables, el marco conceptual del SNI permite formular conjeturas o hipótesis para su examen empírico (Edquist, 2005). desarrollo visto como un proceso de transformación estructural económico y social, compatible con los aportes de la teoría clásica del desarrollo (véase especialmente Hirschman, 1984) que resaltan el papel decisivo de los agentes sociales, políticos y económicos en el proceso de transformación estructural implícito.

El planteamiento teórico se fundamenta, en primer lugar, en la hipótesis central neo-schumpeteriana respecto a las condiciones de desequilibrio permanente en los mercados como consecuencia directa de la incesante actividad innovadora subyacente al desarrollo económico; y, segundo, en la conceptualización de los procesos de generación, difusión y aplicación de conocimiento en la producción como procesos de carácter sistémico, determinados por los vínculos (interacciones) entre tres tipos de agentes genéricos en el sistema económico: empresas, agencias gubernamentales y privadas e instituciones de educación (Arocena y Sutz, 2006).

En los siguientes apartados, a partir de una presentación sucinta de los aspectos más relevantes del marco conceptual del NSI, se delinean los elementos analíticos fundamentales en lo referente al papel de las instituciones respecto a la innovación en el contexto del mundo no industrializado.

\subsection{El Sistema Nacional de Innovación (NSI)}

El marco conceptual del NSI se desarrolla en las últimas décadas de siglo pasado, a partir de los trabajos de tipo apreciativo de Lundvall (1992), Nelson (1993) y Freeman (1987) que, como aporte analítico novedoso, enfatizan en la importancia relativa de los diversos actores sociales en relación con la actividad innovadora y los mercados en los sistemas económicos capitalistas. 
En éstos, el proceso de innovación aparece determinado por las interacciones sistémicas entre empresas, agencias gubernamentales y privadas e instituciones de educación, expresadas en un conjunto más o menos estable e institucionalizado de relaciones entre ellas (Arocena y Sutz, 2002, 2006). Así por ejemplo, Lundvall (1992) destaca la importancia de las relaciones entre productores y usuarios en tanto que definen los espacios interactivos de aprendizaje y las posibilidades de cooperación en la solución de problemas de la producción que devienen en la ampliación de los conocimientos y en el fortalecimiento de las capacidades de innovación.

Un NSI se compone de dos dimensiones claramente definidas e interrelacionadas: en primer lugar, la estructura del sistema, definida por las competencias necesarias para la producción de bienes y servicios diversos de creciente complejidad; y en segundo lugar, el arreglo institucional asociado, referido a la manera como ocurre la producción, la innovación y el aprendizaje en el sistema económico (Lundvall, 1992). Como consecuencia, los procesos de innovación están determinados por las capacidades de los componentes del sistema y sus interacciones.

En relación con la dimensión institucional, que constituye el propósito analítico del presente trabajo, Edquist (2005) señala el carácter no lineal de las relaciones entre actores al interior del NSI, cuya principal implicación es que las empresas no innovan de manera aislada sino que interactúan con otras organizaciones en relaciones complejas caracterizadas por la reciprocidad y los mecanismos de retroalimentación mediante diversos vínculos.

El subsistema institucional es especialmente importante porque constituye el recipiente que permite el desarrollo y la evaluación de la actividad innovadora y el desempeño económico. En este sentido, Metcalfe (2005) define un NSI como: [el] conjunto de instituciones que de manera individual y conjunta contribuye con el desarrollo y difusión de nuevas tecnologías y que proporciona el marco dentro del cual los gobiernos formulan e implementan políticas para influenciar el proceso de innovación (p. 21).

El concepto de institución se define en dos sentidos: en primer lugar, las instituciones son hábitos y reglas de juego $^{4}$ más o menos arraigadas en la sociedad, que desempeñan un papel crucial en la determinación de los modos de interacción y aprendizaje entre individuos, así como en las formas de usar el conocimiento con fines productivos. $\mathrm{Y}$ en segundo lugar, las instituciones son arreglos formales y materializados que definen, por ejemplo, los derechos de propiedad y el funcionamiento de los mercados (Lundvall, 1992).

Si bien en el marco conceptual del NSI no se establece una clara distinción entre ambos, los avances recientes contenidos en la arquitectura analítica de las interacciones micro-meso-macro en el sistema económico (Dopfer, Foster y Potts, 2004) contribuyen, precisamente, en conferirle una dimensión analítica. Consecuentemente, una institución es "un mecanismo de coordinación entre el proceso individual y social de creación de valor económico" (Potts, 2007, p. 342); como tal, son reglas, conocimiento acumulado en el sentido de la primera dimensión expuesta previamente, cuya principal función es contribuir con la reducción de la incertidumbre de los agentes económicos.

De otra parte, el énfasis del papel de las instituciones en los procesos de innovación (Sutz, 1998; Edquist, 2005), hace posible una aproximación a las dinámicas innovadoras en contextos nacionales específicos, en condiciones concretas de relaciones sociales, económicas, políticas y

4 Las instituciones, entendidas como las reglas de juego o el conjunto de hábitos comunes, normas, rutinas, reglas o leyes que regulan las relaciones e interacciones entre individuos, grupos y organizaciones, a la manera de Lundvall (1992). 
culturales especialmente relevantes en países de la periferia capitalista. Por tanto, si las empresas que participan en redes de vínculos con otras firmas y organizaciones en un sistema económico son depositarias de conocimiento, el cual define la variedad de sus comportamientos, las instituciones (reglas) proveen el marco en el que se encuentran imbricados los incentivos, las restricciones y las formas de organización corporativa de cada nación (Sutz, 1998; Edquist, 2005; Cooper, 2006).

\subsection{La innovación y el desarrollo: necesidad de nuevas herramientas analíticas y metodológicas}

Como se ha expuesto, un NSI está definido por el aparato tecno-productivo y el arreglo institucional vigente compuesto de organizaciones (entidades) públicas y privadas e instituciones de educación (Edquist, 2005); el subsistema institucional es la expresión de su capacidad de autoorganización y de las posibilidades de auto-transformación sustentadas en la capacidad innovadora.

La innovación, como elemento endógeno y transformador del sistema económico, se hace viable a través de la implementación de planes de negocios empresariales en permanente competencia, los cuales son el reflejo de las conjeturas acerca de la posibilidad de organización basadas en el conocimiento acumulado hasta el presente (Harvey y Matcalfe, 2005) en un sistema económico.

En las economías de mercado no desarrolladas, en el sentido del rezago relativo en el proceso de industrialización y el escaso fortalecimiento institucional, como sucede en los países latinoamericanos, se reconoce que existe actividad innovadora (Katz, 2006). Sin embargo, ésta ocurre de manera informal; suele ser intersticial, en el sentido de que ocurre con frecuencia en "los márgenes de las princi- pales relaciones de poder económico, político y cultural" (Arocena y Sutz, 2006, p. 2); y, por último, tiene lugar en los escasos circuitos innovadores en los que concurren actores demandantes y oferentes de conocimiento productivo, con una limitada contribución al fortalecimiento de la competitividad de la economía.

Según Arocena y Sutz (2002, 2006), en estos circuitos desempeñan un papel importante los denominados "sastres tecnológicos", pequeñas empresas de base tecnológica que tienen la capacidad de encontrar conocimiento relevante y adaptado a la medida del problema específico, de sus requisitos técnicos, sus restricciones económicas y su contexto social. De hecho, son las células de los NSI, numerosos y variados en el primer mundo, pero que en la periferia no industrializada, de manera contrastante, deben defender su existencia en los intersticios de las relaciones predominantes de poder, con alta probabilidad de perecer.

En atención a estas características, estos autores señalan algunos aspectos del marco conceptual del NSI relevantes en el estudio de los procesos de innovación empresarial que sustentan el cambio estructural en las condiciones específicas de las economías no desarrolladas, a saber:

1. Como concepto relacional pone de relieve las conexiones entre diferentes tipos de actores colectivos, de este modo ofrece un marco general para explorar los procesos concretos de interacción entre actores y organizaciones.

2. Toma en consideración tanto los factores tecno-económicos implicados en los procesos de cambio estructural, como en los temas políticos, institucionales y sociales inherentes. En este sentido, posibilita la descripción de situaciones en las que el conflicto está presente tanto en el ámbito interno del sistema, como en el ám- 
bito macro-social que comprende escenarios diversos como la educación y los espacios de participación y toma de decisiones de los agentes en el sistema.

3. Además del componente positivo, el cual enfatiza en la importancia de las interacciones entre agentes, también contiene un componente político no trivial o normativo que permitiría propuestas estratégicas y de política orientadas a la creación de puentes entre agentes para promover la innovación.

4. Por último, ofrece elementos valiosos para repensar el desarrollo desde un enfoque centrado en la pluralidad de agentes y sus vínculos, no restringido a la dicotomía Estado - mercado.

No obstante, según Jonson y Lundvall (2000) y Arocena y Sutz (2002), las posibilidades de aplicación como herramienta analítica y metodológica en el ámbito de los sistemas económicos no desarrollados se supeditan a algunas adecuaciones.

En primer término, se debe reconocer la inexistencia de una "mejor práctica" o de un "factor clave" respecto a la estrategia de desarrollo. Lundvall (1992) señala la existencia de tres dimensiones institucionales claves de las economías nacionales que pueden incidir de manera diferenciada en las prácticas y los factores de desarrollo: el horizonte temporal, el espacio de la confianza y la racionalidad de los agentes.

Las instituciones definen el horizonte temporal de los agentes en el corto o el largo plazo y contribuyen también con la generación de confianza que define las expectativas respecto a la inconsistencia de los comportamientos, la certeza respecto a la información que se transmite entre agentes que posibilita la asociatividad en el sistema; y, no menos importante, determinan el verdadero alcance de la racionalidad de los agentes, lejos de la racionalidad sustantiva neoclásica que niega la necesidad de instituciones y cercana al concepto de racionalidad comunicativa en el sentido de Habermas (1984, citado en Lundvall, 1992).

Y, en segundo lugar, los NSI en los países no desarrollados, entre ellos los latinoamericanos, son más potenciales que reales; a diferencia del concepto ex post original, de alto contenido empírico, en éstos cobra importancia como concepto ex ante, derivado de la comprensión de la naturaleza sistémica del comportamiento socio-económico con respecto a la innovación, con altas potencialidades analíticas en consideración del carácter fluido de las relaciones entre actores y la flexibilidad de las estructuras organizacionales.

Estas adecuaciones permitirían ponderar convenientemente la relevancia del tejido institucional en dos sentidos: en cuanto a la identificación y caracterización de relaciones bi-direccionales entre técnica e instituciones (Freeman y Lundvall, 1988) que enfatizan en el papel desempeñado por las agencias públicas (gubernamentales) y privadas y por el subsistema educativo respecto a las dinámicas innovadoras; $\mathrm{y}$, acerca de las posibilidades de intervención mediante políticas sistémicas para la innovación, entendidas como instrumentos de articulación entre agentes.

De manera complementaria, desde el punto de vista de una economía no desarrollada, resulta prioritario analizar teórica y empíricamente la interacción entre firmas, entre éstas y las organizaciones públicas y privadas, como variables determinantes de los procesos de innovación y su papel en el desarrollo. Así mismo, interesa un concepto

5 Para los propósitos de este trabajo basta señalar que la racionalidad comunicativa surge de los consensos y la crítica argumentativa mediatizados por el lenguaje y se expresa en el entendimiento entre los individuos. Más que a la adquisición de conocimientos se refiere a la forma en que los sujetos, capaces de lenguaje y de acción, intercambian y hacen uso de él. 
amplio de innovación, a la Schumpeter, que incluya la innovación radical derivada de la investigación y el desarrollo junto a las actividades rutinarias de empresas en sectores tecnológicamente heterogéneos (Johnson y Segura-Bonilla, 2001).

\section{Las interacciones entre} las estructuras micro-mesomacro: un primer esbozo de una propuesta analítica y metodológica en la perspectiva del NSI

\subsection{Características de la estructura analítica micro-meso-macro}

El comportamiento innovador empresarial y las interacciones competitivas en un sector o subsector de la producción, contenidos y articulados en un Sistema de Innovación definido en el ámbito de la nación ${ }^{6}$, aparecen determinadas por las interacciones con otras organizaciones públicas y privadas e instituciones de educación (Carlsson et al., 2002).

Sin embargo, en los países de menor avance industrial el concepto del NSI ha sido empleado principalmente con propósitos descriptivos y en la evaluación prospectiva de los componentes del sistema. En algunos casos se ha propuesto inclusive como paradigma de organización institucional para mejorar la eficacia de la intervención gubernamental respecto a la promoción y mejoramiento

6 Sin embargo, el nivel de definición del sistema puede circunscribirse a lo regional, sectorial y a redes locales de empresas (Carlsson et al., 2002). del desempeño innovador, pero no como una herramienta analítica para el examen profundo de los aspectos relevantes del proceso innovador.

Recientemente, sin embargo, se ha avanzado en el análisis teórico de las interacciones entre los niveles jerárquicos micro-meso-macro del sistema económico con el objetivo de descubrir los elementos característicos y los factores determinantes de la interacción entre las instituciones e innovación. Estos desarrollos complementan el concepto del NSI en tanto que proporcionan una estructura analítica y, especialmente, metodológica, útil en la formulación de hipótesis contrastables respecto al fenómeno de la innovación.

Según Foster y Potts (2007) y Dopfer, Foster y Potts (2004), la estructura analítica micro-meso-macro logra una representación adecuada de la economía como un sistema complejo compuesto de reglas genéricas (conocimiento) y su respectiva población de adoptantes, contenidas en las denominadas unidades meso que sirven de soporte a las interacciones entre los dominios micro y macro.

Conocidas como instituciones, normas, acuerdos, leyes, tecnologías, etc., las reglas genéricas involucran las dimensiones cognitivas, del comportamiento, organizacionales, técnicas, institucionales y socio-culturales que soportan la actividad económica en la medida en que permiten la existencia de comunidades de entendimiento e intercambio (Foster y Potts, 2007). Son esencialmente adaptables, asequibles, transmisibles y transformables por los agentes del sistema y se presentan arraigadas o embebidas en poblaciones estables que las comparten.

Por su parte, dos elementos permiten identificar y caracterizar las variaciones en los ámbitos micro y macroeconómicos: de un lado, la tendencia de una variante de una regla cualquiera a dominar las otras en un proceso que implica la existencia de la variedad; y, por el otro, el con- 
secuente proceso de cambio en la población de adoptantes de las reglas genéricas.

En el marco analítico y metodológico micro-meso-macro, la innovación involucra la generación de nuevas reglas genéricas y la dinámica de la población adoptante en el ámbito micro, junto a sus implicaciones en el nivel macro agregado. En este sentido, el dominio micro se refiere a las elecciones individuales de agentes y organizaciones, y el macro al resultado emergente asociado a ellas (Dopfer, Foster y Potts, 2004).

Como elemento esencial, el cambio en el sistema aparece asociado a la generación continua y espontánea de la novedad, guiada por las propiedades emergentes de adaptación (selección) y auto-organización (Dopfer, Foster y Potts, 2007). El mecanismo de selección, dada la innovación permanente, conduce a que la amenaza competitiva constituya un estímulo para la innovación; por su parte, de acuerdo con el principio de auto-organización, la tendencia hacia el estado estacionario afecta el estado estacionario mismo, lo cual mantiene al sistema económico por fuera de las condiciones ideales de equilibrio.

Consecuentemente, la explicación del proceso de cambio estructural se fundamenta en la dinámica en el nivel meso que está determinada por los procesos emergentes y perfectamente diferenciados de cambio y variedad en las reglas micro (organización) y la emergencia de un patrón auto-organizado de orden expresado en los agregados macro; la simultaneidad de ambas dimensiones analíticas en el tiempo y el espacio configuran la denominada trayectoria meso (Foster y Potts, 2007; Dopfer y Potts, 2004).

De otra parte, la trayectoria meso es la generalización del concepto de trayectoria tecnológica identificada en los estudios sobre innovación (Dosi, 1984) e involucra el proceso de creación-destrucción por el cual se perturba un orden y una organización establecidos mediante la introducción de una nueva idea (regla) en el dominio micro y el crecimiento de la respectiva población de adoptantes o difusión antes de estabilizarse en un nuevo orden y organización en el dominio macro.

La evolución económica o desarrollo, consecuentemente, es el proceso asociado con los cambios en la estructura de reglas genéricas concebido como trayectoria meso en tres fases: primera, la generación de una regla novedosa (innovación); segunda, la adopción de la regla en una población de agentes micro que conforman una población; y, tercera, la retención y uso de reglas meso novedosas en las estructuras micro y macro de la economía.

\subsection{Bosquejo de una propuesta analítica y metodológica}

En este apartado se presenta una primera aproximación a una propuesta analítica y metodológica en el estudio de la relevancia de las instituciones, entendidas como reglas, en el desempeño innovador de las empresas en sistemas económicos de menor desarrollo industrial.

Una vez definido el problema pertinente y relevante a investigar, el cercamiento analítico y metodológico genérico consiste en la caracterización del Sistema de Innovación objeto de estudio y, de acuerdo con Foster y Potts (2007), en la identificación de las características e interacciones de los ámbitos meso, macro y micro.

\subsubsection{Las características relevantes del Sistema de Innovación}

En general, de acuerdo con Edquist (2005), el marco conceptual del NSI puede contribuir con la necesidad de los sistemas económicos menos desarrollados de adelantar 
mejor investigación de las actividades de desarrollo, difusión y uso de conocimiento que confiera mayor alcance explicativo de los procesos de innovación, especialmente mediante estudios comparativos y de caso.

Dada la innovación y los procesos de aprendizaje como propósito analítico, en este marco ésta es concebida como la producción endógena de nuevo conocimiento o la combinación de conocimiento existente resultado de muchos factores y sus retroalimentaciones en un proceso histórico de características variables entre países, que no obedece al desempeño de un sistema óptimo ideal.

Esto implica definiciones precisas, en primer lugar, de los elementos implicados en el sistema tales como actividades productivas, innovación, función, componentes, organización e instituciones; segundo, caracterización de las relaciones entre los agentes en el sistema; y tercero, identificación de las fuerzas determinantes de la actividad innovadora y sus relaciones variables.

\section{Los componentes del sistema y sus relaciones}

El Sistema de Innovación es un todo coherente limitado, con características y metas diferentes a las de sus componentes y que contiene las interacciones entre ellos. Analíticamente, sin embargo, no es necesaria la especificación de todos los elementos y sus características, pues imposibilitaría el estudio del mismo. En su lugar, es viable la inclusión y consideración de subsistemas compuestos por agentes con características y propósitos comunes; esto es, estructuras analíticas jerarquizadas.

El énfasis analítico recae entonces en las interdependencias complejas y no-lineales entre agentes del sistema. Las empresas no son los agentes optimizadores individuales convencionales que innovan de manera aislada, sino que interactúan con otras organizaciones en relaciones com- plejas caracterizadas por la reciprocidad y los mecanismos de retroalimentación mediante diversos vínculos; es decir, los procesos de innovación son influenciados tanto por los componentes del sistema como por las relaciones entre ellos.

Como los principales componentes del sistema se definen las organizaciones y las instituciones. En primer lugar, las organizaciones, actores o agentes, son las estructuras formales creadas conscientemente con un propósito explícito; entre éstas las empresas desempeñan un papel crucial en lo que respecta a la innovación, junto a las agencias públicas y privadas (agencias gubernamentales y agremiaciones privadas) y los entes de educación. Y, en segundo lugar, las instituciones, entendidas como reglas de juego o conjuntos de hábitos comunes, normas, rutinas, reglas o leyes que regulan las relaciones e interacciones entre individuos, grupos y organizaciones.

\section{Las funciones y actividades del sistema ${ }^{7}$}

Las funciones y actividades se refieren a la creación, difusión y explotación de la innovación tecnológica dentro del sistema. Entre las principales actividades en los sistemas de innovación se encuentran: la investigación y desarrollo $(\mathrm{I}+\mathrm{D})$, la implementación o aplicación del conocimiento con propósitos productivos, la educación del recurso humano y el eslabonamiento de las actividades en su interior y con otros sistemas.

En cuanto a las funciones que soportan el crecimiento de las actividades productivas no existe consenso, pero es posible identificar, entre las más importantes, aquellas con la creación de nuevo conocimiento y de oportunidades tecnológicas, el direccionamiento de los procesos de

7 Actividades y funciones que, en todo caso, dependen de los objetivos investigativos. 
investigación, la provisión de recursos y facilitar la formación de los mercados y su regulación, entre otras.

En general se acepta que las actividades son los factores que influyen sobre el desarrollo, difusión y uso de innovación que analíticamente se describe en una trayectoria meso y que su función como soporte del desarrollo es la provisión de recursos para los procesos de innovación (desarrollar, difundir y uso de conocimiento).

A manera de síntesis, el proceso de innovación analizado en este marco es un fenómeno multi-causal que implica establecer la importancia relativa de sus determinantes, sus causas, y que permita establecer un orden jerárquico entre ellos.

\subsubsection{Los ámbitos micro-meso y macro y la trayectoria meso}

La teoría de los sistemas de innovación sirve como marco conceptual en el análisis empírico específico de condiciones concretas de desarrollo innovador en contextos de menor desarrollo industrial, atendiendo a sus particularidades. Subyace a este planteamiento el principio metodológico de que son los individuos y las organizaciones quienes adelantan las actividades con significado económico y que las instituciones (reglas) proveen los incentivos y las limitantes a dichas actividades.

Metodológicamente, interesan las relaciones entre actividades y componentes y entre componentes. $\mathrm{Al}$ respecto, los principios metodológicos adoptados indican que no existe una relación uno a uno entre organizaciones y actividades, aunque la flexibilidad de esta relación debe ser limitada; adicionalmente, que la relación entre actividades e instituciones no es directa o expedita, pero que las últimas influyen de manera decisiva en la forma como las organizaciones desempeñan las primeras; y finalmente, que las relaciones entre componentes pueden ser de mercado o por fuera de él, cuya consecuencia directa es la posibilidad de tratar los procesos de competición y transacción (intercambio) junto a los de aprendizaje y conformación de redes, asociados también a la colaboración y cooperación entre agentes.

El carácter complejo de las interacciones de doble vía entre organizaciones e instituciones, y las relaciones de mutuo reforzamiento o contradicción entre instituciones, subyacentes al proceso de innovación, sugiere el acercamiento analítico y metodológico propuesto en los desarrollos evolucionistas recientes contenidos en la denominada estructura ámbitos micro, meso y macro que se apoyan en métodos de modelación y simulación computacional.

\section{El ámbito y la trayectoria meso}

Como instancia de la adopción y difusión de una regla exitosa por parte de una población de agentes micro, el ámbito meso corresponde a la fase de aprendizaje, cooperación y rivalidad competitiva. El núcleo analítico en este ámbito es la dinámica de la población de agentes y sus interconexiones mediadas por reglas, dinámica que se expresa en la trayectoria meso.

Ésta se identifica y caracteriza mediante el análisis histórico y el empleo de series de datos agregados que proporcionan información acerca de las conexiones claves y de la existencia de reglas genéricas en la entidad económica objeto de estudio.

En una trayectoria meso particular las reglas surgen y desaparecen, los agentes heterogéneos adoptan y adaptan reglas genéricas y, de este modo, determinan su permanencia o desaparición. El propósito analítico consiste en identificar diferentes tipos de reglas genéricas que posibilitan las conexiones entre los componentes del sistema 
generadoras de valor económico, reglas genéricas relativamente estables en el tiempo y que se expresan en leyes, normas tácitas y convenciones de amplia aceptación.

En este proceso complejo de interacción entre lo micro y lo macroeconómico soportado en la existencia de reglas meso ${ }^{8}$ se asume que el valor generado en el nivel microeconómico, de carácter heterogéneo, es separable en dos componentes definidos en un período temporal y en algún nivel de agregación de mayor jerarquía. En primer lugar, el efecto orden, derivado de meso-reglas de amplia aceptación que se manifiestan en los datos agregados, en los comportamientos auto-regresivos (debido a la persistencia de las meso-reglas) y en las tendencias perdurables. Y, segundo, el efecto complejidad, derivado de las interacciones entre reglas micro operacionales heterogéneas, las cuales se conectan jerárquicamente con meso-reglas, pero se desconectan de la periferia del sistema.

En esta dinámica las innovaciones incrementales y los procesos de aprendizaje resultan en micro reglas novedosas en tanto que las más viejas son abandonadas, al mejor estilo de la creación-destrucción propuesto por Schumpeter. Alguna regla micro se convierte en una regla meso en la forma de una institución y, en la dirección opuesta, las reglas micro dependen de la existencia de poblaciones estables de reglas meso.

\section{El nivel macro}

La estructura macro comprende las redes cambiantes entre agentes que comparten conexiones locales, en la proximidad del agente, o de largo alcance, en tanto que involucren otros agentes, muchos o pocos. El ámbito macro corresponde a la instancia de la retención/replica-

8 Proceso que, por cierto, no tiene nada que ver con la micro-fundamentación de los comportamientos agregados pretendida en la corriente teórica dominante en economía. ción y uso/reforzamiento de reglas meso, en el que se encuentran las conexiones clave en el sistema económico, identificables mediante el análisis de la información contenida en series de datos agregados.

A las series temporales de datos macro, las cuales contienen una mezcla de fluctuaciones y tendencias, les subyace un conjunto de reglas genéricas (meso) que condicionan la existencia misma de la estructura económica, y de reglas idiosincráticas (micro) relacionadas con las organizaciones, procesos y productos.

A manera de síntesis, las propiedades estadísticas de las series macro relevantes y sus posibles asociaciones brindan información que puede contrastarse con los hallazgos previos respecto a las reglas meso provenientes del análisis histórico y estudios de caso. En general, las tendencias auto-regresivas deterministas son indicio de la operación de reglas meso, en tanto que la complejidad se asocia a la gran diversidad de reglas micro.

\section{El ámbito de lo micro}

Como se ha bosquejado previamente, el ámbito micro corresponde a la instancia de la generación permanente de nuevas reglas en la forma de innovaciones por parte de los agentes en el sistema (empresas, individuos y organizaciones), muchas de las cuales fracasan al enfrentar el proceso de selección del mercado.

Dado que la heterogeneidad (diversidad) de los agentes es la fuente de generación de valor, se asume que existen reglas específicas operacionales que, conectadas jerárquicamente con meso-reglas genéricas, confieren complejidad al sistema. La fase micro se encuentra relacionada y es dependiente de la meso en la medida en que las reglas específicas micro eventualmente se convierten en reglas meso en la forma de nuevas instituciones que reemplazan las viejas. 
De este modo, el análisis en el ámbito micro tiene como objetivo primordial identificar las motivaciones que dan nacimiento a las organizaciones, productos y procesos en el nivel microeconómico y la manera como éstos devienen en transiciones estructurales que implican cambios en el conjunto de reglas meso.

Desde el punto de vista del método, los modelos de simulación/calibración permiten una aproximación a la manera como los agentes usan las reglas micro y meso como mecanismos para generar valor económico. Así mismo, a partir de los datos históricos, es posible la caracterización general de los agentes involucrados, su comportamiento e interacciones.

\section{Conclusiones}

En esta exploración teórica se ha discutido la relevancia del marco conceptual del NSI y la estructura analítica micro-meso-macro, en la perspectiva de la economía evolucionista moderna, que de manera conjunta brindan herramientas analíticas y metodológicas útiles en la exploración del fenómeno de la innovación y el contexto institucional en economías de menor desarrollo industrial. En el artículo también se describe una metodología genérica para la aproximación al problema de la innovación en contextos institucionales específicos, con fundamento en el concepto de institución como regla de juego de los agentes en los sistemas económicos.

$\mathrm{Al}$ respecto, se encuentra que el marco conceptual del NSI se ha empleado primordialmente como un modelo de arreglo institucional funcional respecto a la formulación e implementación de políticas de fortalecimiento de capacidades innovadoras, pero no como una estructura analítica y metodológica útil en la formulación y contratación de hipótesis pertinentes. Ejemplo de ello son los aportes contenidos en los trabajos sobre Competitividad
Sistémica del Instituto Alemán de Desarrollo (Esser, 1994) y sobre el Diamante Competitivo de Porter (1991) que definen la competitividad de la economía como el resultado de medidas articuladas desde los niveles micro, meso, macro, y meta-económicos y la toma conjunta de decisiones por parte de grupos importantes de agentes en el sistema.

No obstante, en la perspectiva teórica evolucionista, la cual asume la innovación como el fundamento de los cambios estructurales en los sistemas económicos capitalistas, incluidos los menos desarrollados, se encuentra que el marco conceptual del NSI permite escudriñar la naturaleza sistémica del fenómeno, definido por las interacciones de los agentes económicos y sociales actuantes en los aparatos productivo e institucional en permanente transformación, con base en la introducción, difusión, adopción y transformación de reglas.

Adicionalmente, se identifica la coherencia del marco conceptual del NSI con los desarrollos evolucionistas modernos contenidos en la estructura micro-meso-macro que contribuye con la definición de una metodología genérica para el estudio de la innovación centrada en los agentes, las reglas, las interacciones, las características particulares y los cambios en el entorno.

Por último, en contraste con los abundantes estudios centrados en los procesos de transferencia y difusión tecnológica que, en lo fundamental, concluyen en la necesidad del cierre de la brecha o catching-up respecto al mundo industrializado, se identifican trabajos recientes contenidos en el marco de los NSI cuyos resultados, más significativos, identifican "circuitos innovadores" y "espacios interactivos de aprendizaje" como fundamento de la construcción de ventajas competitivas dinámicas a partir del cambio tecnológico en sectores de baja tecnología y la potencialización de recursos ya existentes en las economías no desarrolladas (Arocena y Sutz, 2002, 2006). 


\section{Referencias}

Arrow, K. J. (1979). El bienestar económico y la asignación de recursos para la invención. En N. Rosenberg (Ed.), Ecomomía del cambio técnico (pp. 151-167). México: Fondo de Cultura Económica.

Arocena, R. y Sutz, J. (2006). El estudio de la innovación desde el sur y las perspectivas de un Nuevo Desarrollo. Revista Iberoamericana de Ciencia, Tecnología, Sociedad e Innovación, 7. Montevideo: Universidad de la República. Disponible en <http://www.campus-oei.org/revistaactsi/numero7/articulo01.htm>.

Arocena, R. y Sutz, J. (2002). Innovation Systems and Developing Countries (Working Paper No. 02-05).

Arocena, R. y Sutz, J. (2000). "Looking at National Systems of Innovation from the South", Industry and Innovation, 7, 55-75.

Carlsson, B., et al. (2002). Innovation Systems: Analytical and Methodological Issues. Research Policy, 31, 233-245.

Cooper, C. (2006). Relevance of Innovation Studies to Developing Countries. Disponible en http://www. unu.edu/unupress/unupbooks.

Dopfer, K., Foster, J. \& Potts, J. (2004). Micro-meso-macro. Fournal of Evolutionary Economics, 14, 263-279.

Dosi, G. (1984). Technical Change and Industrial Transformation. Londres: Mac Millan.

Edquist, C. (2005). Systems of Innovations. Perspectives and Challenges. En J. Fagerberg, D. Mowery and R. Nelson (Eds.), The Oxford Handbook of Innovation (pp. 181-208). Oxford, USA: Oxford University Press.
Esser, K., et al. (1994). Competitividad sistémica. Competitividad internacional de las empresas y políticas requeridas (Instituto Alemán de Desarrollo). Disponible en <http://www. meyer-stamer.de/1994/systemsp.htm>.

Foster, J., \& Potts, J. (2007). A Micro-Meso-Macro Perspective on the Methodology of Evolutionary Economics: Integrating History, Simulation and Econometrics (The University of Queensland, School of Economics, Discussion Paper No. 343).

Freeman, C. (1987). Technology Policy and Economic Perfomance - Lessons From Japan. Londres: Pinter Publishers.

Freeman, C. \& Lundvall (Ed.). (1988). Small Countries Facing the Technological Revolution. Londres: Pinter Publishers.

Harvey, M. \& Metcalfe, J. S. (2005). The Ordering of Change: Polanyi, Schumpeter and the Nature of the Market Mecanism (Discussion Paper No. 70). Manchester: University of Manchester - CRIC.

Hirschman, A. (1984). De la economía a la politica y más allá. México: Fondo de Cultura Económica.

Katz, J. (2006). Cambio estructural y capacidad tecnológica local. Revista de la CEPAL, No. 89, agosto.

Johnson, B. \& Lundvall, B-A. (2000). Promoting Innovation Systems as a Response to the Globalising Learning Economy. Artículo presentado en el seminario "Arranjos e Sistemas Produtivos Locais e as Novas Políticas de Desenvolvimento Industrial e Tecnológico", Río de Janeiro.

Jhonson, B. y Segura-Bonilla, O. (2001). Innovation Systems and Developing Countries: Experiences from the SUDESCA Project. DRUID (Working Paper No 01-12). 
Lundvall, B. A. (Ed.). (1992). National Systems of Innovation - Toward a Theory of Innovation and Interactive Learning. Londres: Pinter Publishers.

Nelson, R. (Ed.). (1993). National Innovation Systems. London: Oxford University Press.

Metcalfe, J. (2005). Innovation, Competition and Enterprise: Foundation for Economic Evolution in Learning Economics (CRIC, University of Manchester, Discussion Paper No. 71).

Porter, M. (1990). The Competitive Advantage of Nations. London: MacMillan.

Potts, J. (2007). Evolutionary Institutional Economics. fournal of Economic Issues, 2, (XLI).
Sen, A. (2000). Development as Freedom. New York: Anchor Books.

Schumpeter, J. A. (1989). The Theory of Economic Development. New Brunswick, USA: Transaction Publishers.

Sutz, J. (1998). La caracterización del Sistema Nacional de Innovación en el Uruguay: enfoques constructivos (Nota Técnica 19/98). Instituto de Economia da Universidade Federal do Rio de Janeiro - IE/UFRJ. Rio de Janeiro, Brasil.

Williamson, O. (1975). Mercados y jerarquías: su análisis y sus implicaciones antitrust. México: Fondo de Cultura Económica. 